\title{
Modelling Trust in Semantic Web Applications
}

\author{
Gregory Albiston, Taha Osman, Evtim Peytchev \\ School of Science and Technology \\ Nottingham Trent University \\ Nottingham, UK \\ Email: \{gregory.albiston, taha.osman, evtim.peytchev\}@ntu.ac.uk
}

\begin{abstract}
This paper examines some of the barriers to the adoption of car-sharing, termed carpooling in the US, and develops a framework for trusted recommendations. The framework is established on a semantic modelling approach putting forward its suitability to resolving adoption barriers while also highlighting the characteristics of trust that can be exploited. Identification is made of potential vocabularies, ontologies and public social networks which can be used as the basis for deriving direct and indirect trust values in an implementation.
\end{abstract}

Index Terms-interpersonal trust; semantic modelling;

\section{INTRODUCTION}

Car-sharing, termed car pooling in the United States, has been a feature in many countries for decades with its popularity rising and falling [1, p. 93-94]. Initiatives to encourage car-sharing include dedicated lanes, reduced tolls, parking spaces, pick up points and organised schemes. The benefits of reducing the number of car journeys can be perceived both by the individual, as financial and social [2, p. 9], and by wider society, through reduced congestion, pollution and fuel consumption [1, p. 96].

The arrangement of shared journeys can vary from ad-hoc collection at informal or designated locations through to preplanned organisation via public websites or privately within orginisations and companies. The proliferation of smartphones with internet enabled applications is resulting in a blurring between casual ad-hoc and pre-planned journeys [1, p. 106]. Two potential barriers identified to the promotion and adoption of organised car-sharing in the United Kingdom by [3, p. 16$20]$ is the "real and perceived concerns about personal safety" and inter-operability between service providers to faciliate better matching between participants drawn from a larger pool.

Recomender systems have been developed which provide suggestions between individuals based on similarity profiles and their ratings of items. A focus of research is complementing these recommenders with a social network and deriving the trust relationships between individuals [4]. References to trust in this context are to interpersonal trust between two individuals as opposed to intergroup or computer security and authentication trust.

\section{Motivation}

This paper discusses the context of carsharing and a possible approach to reduce barriers to uptake through the use of semantic modelling of trust recommendations.

\section{A. Motivational Scenario}

Bob regularly commutes to work by car. His journey is repeated daily at the same time with long periods spent in traffic. While the radio helps provide some distraction Bob craves conversation and a relief from the daily monotony of driving. He realises that he recognises many of other drivers who undertake a similar journey each day. However, he doesn't know these people and wouldn't know how to start talking to them about car-sharing. He considers talking to his existing contacts but if he tried to contact each directly it would take time, effort and luck to find and arrange to travel with someone.

Instead Bob uses his car-sharing agent to identify people who are registered to car-share. Recommendations are derived from a semantic knowledge base to find individuals Bob is likely to trust and form a positive relationship and experience. The knowledgebase is supplemented using the registered users' social networks, which includes close friends, work colleagues and their acquaintances, along with collected and other sources of information to derive linkages in interests, personal preferences and relationships. Bob is able to review their travel plans, including route and time of day, to identify with whom he could share. The suggested route to accommodate Bob in the plan, the predicted journey time, cost saving and public transport alternative are made available. Several options are available with one being a close friend. Bob knows his close friend to be unreliable at timekeeping while the others have teh occasional poor feedback or are at the limit of his detour tolerance so he decides to publish his own plan.

A few days later Bob is contacted by the agent to suggest a potential match. The agent informs him that other people have had a good car-sharing experience with the match, have similar routes and both have an interest in cricket. The pair agree to car-share and alternate the driving each day so the cost is shared between them. This removes a car from the road network, reducing both congestion and pollution, while also reducing the travel cost for both parties involved.

\section{B. Considerations and Objectives}

Given the motivational scenario that has been described there are several considerations which can be identified. The area of geographic interest for a participant is likely to be quite small. Only those travelling in the same direction, same time and similar route are of any interest. Yet all other participants have the potential to be interested as new journeys are planned. 
Journeys also occur over a geo-graphically continuous area. A participant with a long distance route between two cities could be of interest to a participant who is only crossing one of the cities if their destinations are identical. Therefore, partitioning and pre-filtering of participants has to be carefully considered to avoid excluding those who have valid journeys.

The relationships and trust between individuals is volatile and constantly changing. Participants will join and leave the network. New relationships will be formed and their nature changed. Personal information will be added, excluded or adjusted. In addition, an active and successful car-sharing group will see participants form bi-directional relationships with each other as they travel together. For example, participants travelling to the same workplace would form a highly clustered and cyclic graph of relationships. Therefore, any traversal of the social network will have to take into account that paths between two participants can be numerous, meandering and subject to change.

Interoperability between alternative systems [1, p. 107] through technology, along with multi-modal integration of options and costs, could help overcome the "critical mass barrier, which has limited the potential of this mode in the past" implies that scalibility is an additional factor. Therefore, complex real-time traversal and calculation could be impractical. Yet, pre-computation could result in significant redundant data that is never utilised as participants can also be geographically dispersed with no interest in each other.

Concerns about personal safety have been characterised by public information campaigns warning against the danger in accepting lifts from strangers, insurance and litigation liabilities and concern about sharing too much personal information with strangers, even in private schemes [3, p. 16]. Information clarifying the legal stand point and good practices in personal safety are widely used to enable participants to safeguard themselves. Efforts have also been made to encourage trust between participants by websites introducing community feedback ratings and leveraging public information on social networks to provide a more informed choice [3, p. 17].

Yet, the provision of this information is still reliant upon the participant investigating each option and making a reasoned selection, based on their own informed or uninformed criteria, from a host of possible candidates. Choosing to car-share means, for one or more participant, a decrease in autonomy and increased time investment when compared to driving alone [2, p. 9]. Repeatedly experiencing poor quality car-sharing following lengthy searches would therefore be a further deterrent. In addition, more and accurate personal information will assist a user derived selection but contradicts concerns about over sharing information with strangers. The realisation of it's wide accessbility could lead to removal and make the perfect match indistinguishable from the obfuscating malefactor.

Therefore, there is added value in a framework that can analyse both geographical and secured personal information to provide a short list of relevant and trusted recommendations for final user selection. Inherent in this concept is inferring the level of trust that can exist between two participants. Participants are likely to have direct contact with relatively few participants in the whole car-sharing scheme. Therefore, matching new or unfamiliar participants will require some form of trust estimation.

Based upon these considerations the following objectives have been indentified:

- Interoperability between car-sharing groups.

- Scalability both for geography and number of participants.

- Able to handle a volatile and evolving social network.

- Wide ranging appropriate recommendations that encourage confidence in the compatiblity of the selection.

\section{RELATED WORK}

There is a body of work relating to both car-sharing and trust derived from social networks. Discussion relating to trust is covered in later sections. This section identifies relevant work on recommending journeys for car-sharing using social network approaches.

The algorithm developed in [5] prioritises journeys based on the existence of past shared journeys, feedback between users and fully or partial completion of the journey. The work of [6] describes a cloud-based multi-modal transport planner including car-sharing. Social network information is used to identify matches, based on friends and friends of a friends, prioritised by minimising journey detour. Similiarly the system described by [7] seeks to arrange "instant" car-sharing and use the "strength of social connection" through mutual friendship to prioritise alternatives. The system developed by [8] allows users to create "pre-arranged crews" and specify for matching individuals they prefer or dislike.

Each of these approaches utilises specific aspects of trust and are constrained by existing relationships, to one degree of seperation, within the social network. They do not seek to identify potential relationships or implicit trust that could be used to encourage car-sharing between stangers.

\section{Semantic Modelling}

The Semantic Web provides a framework for information to have "well-defined meaning, better enabling computers and people to work in cooperation" [9, p. 3]. A semantic approach to modelling seperates the meaning and interactions of information in the domain from the data in a particular instance. Rather than the structure of the data describing the inter-relationships, a seperate ontology provides the structure and relationships while the data can be held as a collection of unstructured triples.

This seperation provides a number of distinct advantages that can be exploited. The ontology can be modified and extended without affecting the underlying data. Therefore, relationships and meaning can be added and removed without risk of compromising the data. The expressivity available in describing ontologies, through concepts and sophisticated relationships such as cardinality and jointness [10, p. 2], enables knowledge to be integrated into the model upon 
which automated reasoning can take place. This enables the inferrence of facts not explicitly stated, such as relationships between individuals. e.g. The grandparents of an individual can be inferred based simply on the explicit facts of individual's parentage.

Ontologies can be shared publicly and re-used either to exploit and navigate a data source or to contribute to the ontology of a new model. This exploitation enables disparate data sources to be interrogated and consumed without the need for knowledge of the design decisions that take place when structuring data, such as applying normalisation. The exploitation of external data sources enables the knowledgebase within a system to be enriched further with the retrieval of additional data not available in the original knowledgebase. Public encyclopedia information and facts can be retrieved from services such as DBpedia [11] and GeoNames [12]. It has been highlighted that interoperability between car-sharing providers along with multi-modal integration will provide added value to the industry and customers. Therefore, the application of a semantic modelling approach is in keeping with these objectives. Further, as the Semantic Web expands this approach enables the potential for the knowledgebase to be used as a source of information.

An underlying concept of a car-sharing scheme is the social network which develops between participants. A representational form of social networks is a directed graph (node, edge, node) which directly aligns with the triple (subject, predicate, object) format used in semantic modelling. Therefore, intuitive descriptions of a social network can be directly translated into an ontology and underlying data set of triples.

\section{A. Defining The Domain}

Car-sharing has existed in various forms since the 1940s [1]. Given its variety it is vital a clear and focussed definition is developed as the basis of the model.

In the UK car-sharing or car sharing [3, p. 22], termed carpooling in the US as well as peer-to-peer car-sharing, liftsharing and journey-sharing, is an individual with a private vehicle allowing others to temporarily utilise the spare passenger seating during a journey. In the US carsharing, termed car clubs in the UK, is the short term "use through hourly rates and subscription-access plans" by members to access a fleet through self-service collection and return to designated parking areas [13]. The area of concern will be the former with one, some or all participants privately owning a vehicle.

There are also different forms of car-sharing. One form has casual, ad-hoc and impromptu arrangement of car-sharing at formal or informal locations, termed "slugging" in the US [1, p. 101]. A driver will collect their passenger(s) from the front of a queue and deliver to a pre-determined destination, often a major employer or central urban location. Mobile applications are opening up the possibility for even the point of collection and destination to become ad-hoc with drivers and passengers being matched prior to or in transit [1]. Alternatively, public websites and private schemes enable the pre-planning of journeys. Participants publish a planned route in advance which

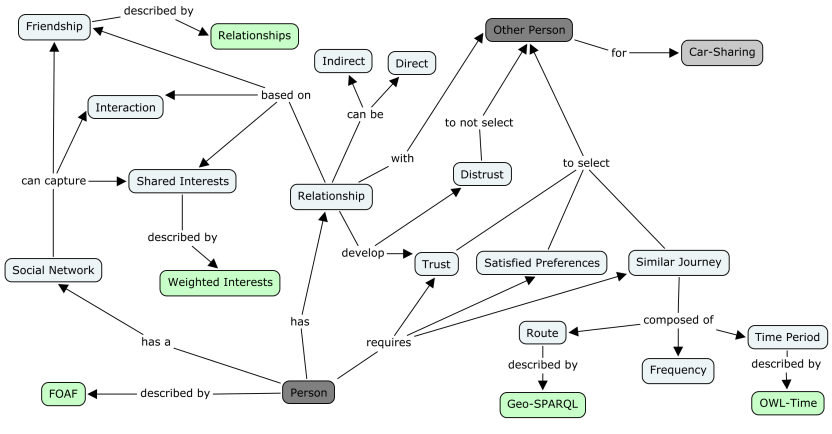

Fig. 1. Concept Map

other participants can view and join. Frequency can range from one-off to a regular daily commute. The latter will be the area of concern with the forward planning of journeys. Both forms can establish a community with centralised or self regulation.

Figure 1. shows a conceptualisation of car-sharing with focus on interpersonal trust. It includes references to standard and pubilicly available ontologies and vocabularies that can be utilised to both enhance the design but also enable the potential for consuming additional data sources. The notion is captured of a Person having a Relationship with Other Person, which defines Trust and Distrust between them. Relationships can be Direct or Indirect and are based upon Friendship, Interaction and Shared Interests. The basis for these concepts will be expanded further in the following section. Also, captured are the concepts of a Simliar Journey and Satisfied Preferences.

Trust is one of several factors considered in the process of selecting a shared journey incomparison to alternatives. The spatial and temporal alignment of both participants presents the concept of a Similar Journey. Disalignment leads to dissatisfaction or immediate rejection when beyond an individuals tolerance. A journey across London is not relevant to a commute across Edinburgh while a journey on a Monday is not relevant to travelling on a Friday. Yet, a journey that adds two minutes of travel is more interesting than a journey that would add five minutes but ten minutes is unacceptable.

Individual also have personal preferences and characteristics, such as smoking and gender, that need to be satisfied. An individual can trust another completely but through preference would not wish to travel with them, for example if they were a smoker. Similarly, an individual may wish to travel only with their own gender for cultural or perceived safety reasons. These personal preferences would exclude another participant from consideration regardless of all other factors. In this way trust and spatial alignment could be considered to be relative comparison factors while personal preferences and temporal alignment are absolute.

\section{Modelling Trust}

The concept of trust and it's definition is highly dependent upon context [14, p. 30] but interpersonal trust has been defined as the commitment "to take the ambigious path if we believe that the trusted person will take the action that will 
produce the good outcome" [14, p. 33]. The determination of trust between individuals can be based upon a range of factors such as past experience, impartiallity, expertise, rumour or personal history $[15$, p. 2] [14, p. 30].

\section{A. Properties of Trust}

Given the focus of interpersonal trust within social networks several properties can be identified [14, p. 34]:

1) Transitivity \& Propagation: Interpersonal trust can be considered to be broadly transistive as an individual can place trust in another's trust of a third party (A trusts B and $B$ trusts $C$ therefore $A$ should trust $C$ ). This enables the meaningful inference of trust through propagation along a chain of relationships. Yet, the trust will deteriorate as the chain becomes longer.

2) Asymmetry \& Directed: The trust placed in another is not necessarily reciprocated by them. Individuals perception and recollection of events and behaviour can be different. The type, and even existence, of a relationship between them can be perceived differently. The trust placed in another does not imply or guarantee equal trust in return.

3) Relative Value: Trust is not an absolute metric but subject to gradation. Comparison between two individuals would distinguish that one is more trusted than another.

4) Personalisation: Given the personal factors that inform an assessment of trust it is logical that two individuals will have a different view on the level of trust to place in a third individual. Therefore, the known trust of one individual cannot be used as a proxy for another.

5) Domain Specific: The context within which an individual is considering their trust informs their assessment [16]. An individual who is an expert in a particular field would be more trusted on that topic than when they have no experience. Therefore, the direct comparison between trust values from different domains should be treated with caution.

6) Distrust: The opposite of trust is not the absence of trust but it's antonym distrust. This has different characteristics which can affect the consideration of trust. Distrust is not transitive (A distrusts $\mathrm{B}$ and $\mathrm{B}$ distrusts $\mathrm{C}$ but $\mathrm{A}$ could trust C) $[17$, p. 1] but can disrupt the transitivity of trust (A trusts $\mathrm{B}$ and $\mathrm{B}$ distrusts $\mathrm{C}$ so $\mathrm{A}$ distrusts $\mathrm{C}$ ). Distrust differs from trust in its development through a "slow-positive, fast negative dynamic" [18, p. 17]. A single experience can result in distrust while many poisitive experiences are required to build trust.

7) Composability: Indirect trust can be based upon the trust of a number of people, or second opinions (A trusts D because $\mathrm{B}$ and $\mathrm{C}$ do). It would then follow that the neighbours who have direct trust of an unknown individual can contribute to inferring indirect trust. High levels of trust from a number of sources would give greater confidence that an individual can be trusted, even if they are distrusted by one. This gives rise to the notion of malevolent and benevolent individuals who are viewed at an extreme by the vast majority. Yet, given the transitive characteristic of trust one individual could provide a gateway to trusting a malevolent individual (A trusts $\mathrm{B}$ and $\mathrm{B}$ trusts $\mathrm{C}$ but nobody else does).

\section{B. Global and Local Trust}

Two perspectives have been take to trust in recommender systems, global and local. A global value is a single trust value for each individual, a community rating, while a local value exists between each pair of individuals. The global approach simplifies the trust network both in terms of quantity of data and assumptions in relationships. Yet this simplification aggregates away contextual information, such as the clustering common in a social networks [19] [18, p. 5] or extreme trust values between specific individuals. It assumes that the majority trust opinion is true for all and is not in keeping with personalisation property of trust identified earlier.

In contrast a local approach can retain the context but means that there are two types of trust value, direct and indirect. The global approach has been identified in literature as "often not appropriate" for recommender systems [18, p. 4] and so a local approach will be the focus of inquiry.

\section{Direct Trust}

The direct trust value represents the trust that exists between two individuals. Various values have been used in literature, boolean, unit interval or integer, as the basis for inferring trust but often without an explicit explanation of how it can be derived.

The approach of [16] is based on five criteria (expertise, experience, impartiality, affinity and track record), defined by the Hoonoh ontology. Focussed upon word of mouth recommendations it highlights that the domain is important to the trust value. Given it's general focus on word of mouth the merits of this approach are constrained by only three of the five criteria having immediately apparent sources of data within the single domain of carsharing.

This section will explore the rationale provide by [20] in the context of the car-sharing model. Their Direct Trust value is a unit interval, ranging from total distrust to total trust, summation of three equally weighted components.

1) Friendship Trust: This value represents the relationship that exists between two individuals. It's presence signifies the existence of direct trust and is asymmetric while its absence would require the inferrence of an indirect trust value. The FOAF (Friend Of A Friend) vocabulary [21] provides a structure for describing indviduals. The vocabulary provides the simplest representation of a relationship between individuals, knows, which does not capture the relative value of trust. Edges between two individuals that are friends should have a higher trust value than those who are enemies. [20] suggest a methodology using the Relationship vocabularly [22], which extends FOAF knows, to describe a wide variety of relationships and group them into categories, captured within the ontology by each relationship being a $r d f s$ :subPropertyOf of the corresponding category.

A car-sharing system could determine the relationships between users in several ways:

- Directly asking an individual to identify their relationship with other individuals signed up to the scheme. 
- Inferrence through provided personal information. e.g. two people who have the same employer could be considered to be Acquaintance Of, Colleague Of or Works With.

- Leveraging access to public social networks, such as Facebook, to examine explicit connections.

- Car sharing individuals would be inferred to have a minimum relationship of Has Met or Knows In Passing.

The Facebook Graph API [23] provides access, with user authorisation, to several methods for accessing both aquaintances and relationships. An entire list of a user's friends can be retrieved or the specific relationship of a user to family members. Specifying another user allows confirmation of friendship or a list of mutual friends. Specific lists can also be obtained in user created and defined types, such as family, close friends, acquaintances, education, work, current city and restricted. Education and employment information can also be accessed to allow inferring of non-explicit connections.

2) Interests Similarity Trust: Individuals with the same interests are likely to have greater trust than those who dont based on the concept that this expresses similarity between individuals; used as the basis in recommendation systems [24]. Individuals are interested in a number of subject topic and the overlap between them can indicate the similarity.

Determining this value will require gathering directly from individuals or exploiting social networks for stated interests. However, given that a populations interests, and description of those interests, can be quite diverse devising a robust short list that is broad enough to capture all interests but narrow enough for meaningfully comparison could be challenging.

Alternatively, an ontology could be utilised to classify and inference between types of interests. A participant interested in football and another interested in cricket have a common interest of sport. Using a semantic model this inferencing could be carried out externally to the main car-sharing model. The FOAF vocabulary [21] provides for the description of a person's interests and so could enable retrieval of this information where FOAF profiles have been created. The Weighted Interests vocabularly [25] expands upon FOAF by providing basic concepts to capture the temporal nature and significance of an individuals interests.

The Facebook Graph API [23] provides information on user's interests, favourite athletes, favourite teams, political views, religion and people subscribed. All but the last are content pages which in turn identify the "best page" for that concept or topic. Therefore, alignment could be sought between two strangers based on intersecting best pages.

Based on the examined literature, it is not clear whether consideration should be given to an individual's disinterests and further exploration is needed. In a car-sharing scenario a long journey with a person who has a passionate interest which you dislike could impinge upon all your common interests.

3) Interactions Trust: The interactions that an individual experiences with another individual can determine the trust between them. Positive interactions increase trust while negative interactions result in distrust. This value can be derived in a car-sharing system by enabling feedback between carsharers. The value could vary between an overall "star" rating through to specific feedback areas such as timeliness, communication, manner, friendliness and willingness to repeat the carshare.

However, few feedback opportunities could occur between individuals. Repeated travel plans do not coincide while carshares who regualr commute are unlikely to want to provide feedback more than once. Therefore, collecting feedback on different aspects may provide more depth than a single overall rating. Frequent communication between individuals and the progagation of messages within public social networks could provide an alternative source as in [26], although the domain specific nature of trust would need to be considered along with the burden of refreshing values.

The presence of an interaction would infer that a minimum relationship exists between two individuals even if one is not explicitly stated. Therefore, a participant providing feedback on another would enable a level of direct trust to be established.

\section{Indirect Trust}

When a direct relationship does not exist it is necessary to infer an indirect trust value. [27] build upon their earlier work with direct trust [20] by proposing an alogrithm for indirect trust based upon the "Most Trusted Path" (MTP). Each path between the origin and sink is assigned a strength based on the direct trust between nodes. Paths with long chains or low direct trust values, signifying distrust, are rejected. The indirect trust value is the product of the calcuated MTP strength and the direct trust between the last node in the path and the sink. Therefore, the trusted neighbour's direct value is moderated by the quality of the path.

Although, this approach considers various characteristics of trust it has a significant drawback in that determining all paths between origin and sink is intensive. Social networks can become clustered with cyclic relationships resulting in a large number of potential paths. Pre-computation of each node to every other node would generate a significant quantity of redundant data while any change to the underlying direct trust values would prompt a refresh search for paths.

An alternative proposed by [17] applies a probablistic method to postulate the existence of a path between origin and sink with a seperate spring-embedding layout alogrithm. The latter provides a spatial dimension with directly connected nodes being attracted or repelled from each other based on their trust values. Trusted friends are drawn together and enemies pushed apart. An indirect trust value is inferred based on the probability of a path existing between origin and sink and their distance. The spring embedding algorithm impicilty has the desired "transitivity and conflict resolution properties" and scalability to handle very large datasets. [17, p. 3] However, it is noted that the spring-embedding alogrithm presumes a symmetric relationship exists between nodes when a characteristic of trust is asymmetry and that "two nodes may be placed closer together by chance". 
[4] put forward a matrix factorisation technique as a social network recommender. Taking a model-based approach that considers both the similiarity between user's item ratings and their social connections. The use of social connections aims to overcome "cold start users" who have few item ratings while also using trust propagation through direct neighbours. The focus of this work is based around item ratings that do not directly translate to car-sharing and does not consider distrust.

Each of these approaches is distinctly different in how it determines an indirect trust value and report high accuracy. Further work is required to fully examine how they can be effectively adapted to a car-sharing social network while providing acceptable performance. Exploration is also required of whether semantic modelling could both capture direct trust and derive indirect trust value through reasoning and inference. A direct traversal of the graph could present problems already discussed but further development of the semantic model and research could identify characteristics, such as social network clustering and the properties of trust, that can be exploited.

\section{CONCLUSION AND FURTHER WORK}

In this paper, we identified some of the potential benefits and barriers to the real world use case of car-sharing. We have described how a semantic modelling approach could assist in overcoming the barriers of inter-operability and personal safety concerns by a putting forward a framework for recommending journeys based in part on interpersonal trust in a social network. The framework highlights the properties of trust and distrust in interpersonal relations and the key concepts.

It shows how a social network can be further developed through operation of the car-sharing system and that direct trust values can be derived with varying amounts of information from the user. Usage of external information sources, such as public social networks, and standard vocabularies have also been highlighted to show how additional information can be brought into the knowledge base or utilised for other purposes. Several approaches to inferring indirect trust for unknown recommendations, utilising different properties of trust within a social network, have been identified with a view to determining the direction of future work.

\section{REFERENCES}

[1] N. D. Chan and S. A. Shaheen, "Ridesharing in north america: Past, present, and future," Transport Reviews, vol. 32, no. 1, pp. 93-112, 2012.

[2] S. B. DeLoach and T. K. Tiemann, "Not driving alone: Commuting in the Twenty-first century," Citeseer, Tech. Rep., 2010.

[3] C. R. S. W. Group, "Where is 2+ car sharing headed? A review of the journey sharing sector and opportunities for future development," http://www.carplus.org.uk/resources/reports/research-and-reports/.

[4] M. Jamali and M. Ester, "A matrix factorization technique with trust propagation for recommendation in social networks," in Proceedings of the fourth ACM conference on Recommender systems. ACM, 2010, pp. 135-142.

[5] M. Collotta, G. Pau, V. M. Salerno, and G. Scata, "A novel trust based algorithm for carpooling transportation systems," in Energy Conference and Exhibition (ENERGYCON), 2012 IEEE International. IEEE, 2012, pp. 1077-1082.

[6] S. Di Martino, R. Galiero, C. Giorio, F. Ferrucci, and F. Sarro, "A matching-algorithm based on the cloud and positioning systems to improve carpooling." in $D M S, 2011$, pp. 90-95.
[7] G. Gidófalvi, G. Herenyi, and T. Bach Pedersen, "Instant social ridesharing," in Proceedings of the Fifteenth World Congress on Intelligent Transport Systems, Nov 16-20, 2008, New York, NY, USA. Intelligent Transportation Society of America, 2008, p. 8.

[8] M. Bruglieri, D. Ciccarelli, A. Colorni, and A. Luè, "Poliunipool: a carpooling system for universities," Procedia-Social and Behavioral Sciences, vol. 20, pp. 558-567, 2011.

[9] T. BERNERS-LEE, J. HENDLER, O. LASSILA, and E. Meaning, "The semantic web."

[10] T. Osman, S. Rmaswamy, S. Mahmoud, and M. Saeed, "Utilising semantic technologies for decision support in dementia care," in Computer Modelling and Simulation (UKSim), 2013 UKSim 15th International Conference on. IEEE, 2013, pp. 628-633.

[11] J. Lehmann, R. Isele, M. Jakob, A. Jentzsch, D. Kontokostas, P. N. Mendes, S. Hellmann, M. Morsey, P. van Kleef, S. Auer, and C. Bizer, "DBpedia - a large-scale, multilingual knowledge base extracted from wikipedia," Semantic Web Journal, 2014.

[12] GeoNames, "The GeoNames geographical database covers all countries and contains over eight million placenames that are available for download free of charge." http://www.geonames.org/, Feb 2014

[13] S. A. Shaheen, A. P. Cohen, and M. S. Chung, "North american carsharing," Transportation Research Record: Journal of the Transportation Research Board, vol. 2110, no. 1, pp. 35-44, 2009.

[14] J. A. Golbeck, "Computing and applying trust in web-based social networks," 2005.

[15] T. Heath, E. Motta, and M. Petre, "Person to person trust factors in word of mouth recommendation," 2006.

[16] T. Heath and E. Motta, "The Hoonoh ontology for describing trust relationships in information seeking," Personal Identification and Collaborations: Knowledge Mediation and Extraction (PICKME2008), 2008.

[17] T. DuBois, J. Golbeck, and A. Srinivasan, "Predicting trust and distrust in social networks," in Privacy, security, risk and trust (passat), 2011 ieee third international conference on and 2011 ieee third international conference on social computing (socialcom). IEEE, 2011, pp. 418-424.

[18] F. E. Walter, S. Battiston, and F. Schweitzer, "Personalised and dynamic trust in social networks," in Proceedings of the third ACM conference on Recommender systems. ACM, 2009, pp. 197-204.

[19] J. Golbeck, B. Parsia, and J. Hendler, Trust networks on the semantic web. Springer, 2003.

[20] S. Hamdi, A. L. Gancarski, A. Bouzeghoub, and S. B. Yahia, "IRIS: a novel method of direct trust computation for generating trusted social networks," in Trust, Security and Privacy in Computing and Communications (TrustCom), 2012 IEEE 11th International Conference on. IEEE, 2012, pp. 616-623.

[21] D. Brickley and L. Miller, "Foaf vocabulary specification," http://xmlns.com/foaf/spec/, Jan 2014.

[22] I. Davis and E. Vitiello, Jr., "RELATIONSHIP: A vocabulary for describing relationships between people." http://vocab.org/relationship/.html, Feb 2014.

[23] Facebook, "The Graph API - the primary way for apps to read and write to the Facebook social graph." https://developers.facebook.com/docs/graph-api/, Feb 2014.

[24] J. Golbeck and J. Hendler, "Filmtrust: Movie recommendations using trust in web-based social networks," in Proceedings of the IEEE Consumer communications and networking conference, vol. 96. Citeseer, 2006.

[25] T. W. I. Vocabulary, "The weighted interests vocabulary specification provides basic concepts and properties for describing describing preferences (interests) within contexts, their temporal dynamics and their origin on/for the semantic web." http://smiy.sourceforge.net/wi/spec/weightedinterests.html, Feb 2014.

[26] S. Adali, R. Escriva, M. K. Goldberg, M. Hayvanovych, M. MagdonIsmail, B. K. Szymanski, W. A. Wallace, and G. Williams, "Measuring behavioral trust in social networks," in Intelligence and Security Informatics (ISI), 2010 IEEE International Conference on. IEEE, 2010, pp. 150-152.

[27] S. Hamdi, A. Bouzeghoub, A. L. Gancarski, and S. B. Yahia, "Trust Inference Computation for Online Social Networks," in Trust, Security and Privacy in Computing and Communications (TrustCom), 2013 12th IEEE International Conference on. IEEE, 2013, pp. 210-217. 